\title{
A NONTRIVIAL $T_{1}$-SPACE ADMITTING A UNIQUE QUASI-PROXIMITY
}

\author{
by HANS-PETER A. KÜNZI and STEPHEN WATSON $\dagger$
}

(Received 7 December, 1994)

\begin{abstract}
We construct a $T_{1}$-space that is not hereditarily compact, although each of its open sets is the intersection of two compact open sets. The search for such a space was motivated by a problem in the theory of quasi-proximities.

1. Introduction. Answering a question of [3] the first author showed in [4] that a topological space $X$ admits a unique quasi-proximity if and only if its topology is the unique base that is closed under finite unions and finite intersections. (Here we assume that $\cap \varnothing=X$.) Such spaces are studied under the name upq spaces in $[\mathbf{7}, \mathbf{8}]$ and in $[3]$ it is observed that each uqp Hausdorff space is finite (compare also [1, Proposition 4]). Two simple countable examples of first-countable uqp $T_{0}$-spaces that are not hereditarily compact (see for example [9]) are provided in [4]. In [5] it is observed that each uqp $T_{1}$-space in which each point is a $G_{\delta}$-set is hereditarily compact. Similarly, locally hereditarily Lindelöf uqp $T_{1}$-spaces are shown to be hereditarily compact in [2]. These results have led to the question whether each uqp $T_{1}$-space is hereditarily compact $[2,6]$. This simply stated problem has turned out to be very intractable. It is the aim of the present note to show that indeed a uqp $T_{1}$-space need not be hereditarily compact. Our example is scattered (i.e. each nonempty subspace contains an isolated point) and has the property that each of its open sets is the intersection of two compact open sets. The construction is carried out in ZFC, but the cardinality of the obtained space is very large. Curiously, it may be the largest space so far constructed in topology, without the use of additional set-theoretic axioms, to answer a specific question. The problem remains open whether each uqp $T_{1}$-space of small cardinality, say $2^{\omega}$, may be hereditarily compact.
\end{abstract}

2. The example. We begin with a technical result that can be used to derive many known facts about uqp spaces. It will motivate the subsequent construction of the counterexample by showing how close uqp spaces come to being hereditarily compact. Recall that a subset $G$ of a topological space $X$ is called bounded in a subset $Y$ of $X$ provided that each open collection in $X$ that covers $Y$ has a finite subcollection covering G.

Proposition 1. Let $X$ be a uqp space that is not hereditarily compact and let $Y$ be a maximal element among the noncompact open subspaces of $X$. Then for any open subspace $G$ of $Y$ we have that either $Y \backslash G$ is compact or $G$ is bounded in $Y$.

Proof. Let $\mathcal{U}$ be an open cover of $Y$ without a finite subcollection covering $G$. Then

† This paper was written while the first author was supported by the Swiss National Science Foundation under grant 21-30585.91 and by the Spanish Ministry of Education and Sciences under DGICYT grant SAB94-0120.

During his visit to the University of Berne the second author was supported by the first author's grant 21-32382.91 from the Swiss National Science Foundation and by the Natural Sciences and Engineering Research Council of Canada. 
by [4, Proposition] for an arbitrary ultrafilter $\mathscr{G}$ on $X$ containing $\{G \backslash U: U \in \mathscr{U}\}$, there exists a finite (possibly empty) collection $\mathcal{M}$ of open sets in $X$ such that $\cap \mathcal{M} \subseteq \mathrm{G}$ and each member $M \in \mathcal{M}$ contains a cluster point of $\mathscr{G}$. Thus for any $M \in \mathcal{M}$, we have $M \backslash Y \neq \varnothing$, and consequently $Y \cup M$ and $Y \backslash M$ are compact. We conclude that $Y \backslash \mathcal{M}$ and thus also $Y \backslash G$ are compact.

We next define a transfinite sequence of cardinals by using essentially the well-known beth function. Set $\lambda_{0}=1$ and $\lambda_{1}=\omega$. Furthermore for any ordinal $\beta \neq 0$ set $\lambda_{\beta+1}=2^{\lambda_{\beta}}$. Finally, set $\lambda_{\beta}=\sum_{\beta^{\prime}<\beta} \lambda_{\beta^{\prime}}$ for any limit ordinal $\beta$.

LEMma 1. There is an ordinal $\alpha$ with uncountable cofinality such that $\alpha=\sum_{\beta<\alpha}\left(\lambda_{\beta}\right)^{\omega}$.

Proof. Define a class function $f: O n \rightarrow O n$ on the class $O n$ of ordinals by setting $f(\gamma)=\sum_{\beta<\gamma}\left(\lambda_{\beta}\right)^{\omega}$ for each ordinal $\gamma$. Clearly $f(\gamma) \geq \gamma$ for any ordinal $\gamma$ and $f$ is continuous; i.e. for every limit $\gamma, f(\gamma)=\lim _{\delta \rightarrow \gamma} f(\delta)$. Define now a transfinite sequence $\left\{\alpha_{\delta}: \delta \leq \omega_{1}\right\}$ of ordinals as follows. Set $\alpha_{0}=\omega$ and $\alpha_{\delta+1}=f\left(\alpha_{\delta}\right)$ for any ordinal $\delta<\omega_{1}$. Furthermore let $\alpha_{\delta}=\sup _{\gamma<\delta} \alpha_{\gamma}$ for any limit ordinal $\delta \leq \omega_{1}$. Since $f$ is continuous, we see that $f\left(\alpha_{\omega_{1}}\right)=\alpha_{\omega_{1}}$. Because of the continuity of $f$ it is also readily checked that $\alpha_{\omega_{1}}$ has uncountable cofinality.

As usual, for any set $A$, we let $[A]^{\omega}=\{B \subseteq A:|B|=\omega\}$. Here $|B|$ denotes the cardinality of $B$.

We are now going to construct the space described in the introduction.

EXAMPLE 1. Let $\alpha$ be the ordinal $\alpha_{\omega_{1}}$ defined in the proof of Lemma 1. We shall construct inductively a scattered space $X$ of height $\alpha+1$. For any ordinal $\beta \leq \alpha, X_{\beta}$ denotes the set of the isolated points of $X \backslash \bigcup_{\gamma<\beta} X_{\gamma}$. For any point $x \in X$, its level $l(x)$ is the unique ordinal $\gamma$ such that $x \in X_{y}$. The height of a subset $A$ of $X$ is the ordinal $\sup \{l(x)+1: x \in A\}$.

Let $X_{0}$ be a countably infinite discrete space. Consider $\beta \neq 0, \beta<\alpha$ and suppose that the space $\bigcup_{\gamma<\beta} X_{\gamma}$ has been defined inductively such that the following conditions are satisfied.

When having created a level set $X_{\gamma}$ we list $\left[X_{\gamma}\right]^{\omega}$ by indexing it by an initial segment

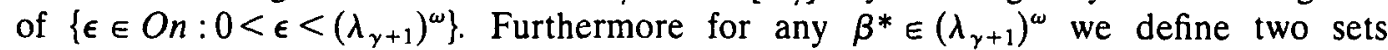
$R^{p}\left(\gamma+1, \beta^{*}\right)$ and $R^{q}\left(\gamma+1, \beta^{*}\right)$ as follows. Assume that $R\left(\gamma+1, \beta^{*}\right)$ is the $\beta^{*}$ th countably infinite subset of $X_{\gamma}$. We write $R\left(\gamma+1, \beta^{*}\right)$ as the disjoint union of two infinite sets $R^{p}\left(\gamma+1, \beta^{*}\right)$ and $R^{q}\left(\gamma+1, \beta^{*}\right)$. In the case that $\beta^{*}$ is not used for listing, we set these two latter sets equal to the empty set.

Moreover for any limit ordinal $\gamma$ we list $\left[\bigcup_{\gamma^{\prime}<\gamma} X_{\gamma^{\prime}}\right]^{\omega}$ by an initial segment of $\left\{\epsilon \in\right.$ On: $\left.0<\epsilon<\left(\lambda_{\gamma}\right)^{\omega}\right\}$. Furthermore for any $\beta^{*} \in\left(\lambda_{\gamma}\right)^{\omega}$ we define two sets $R^{p}\left(\gamma, \beta^{*}\right)$ and $R^{q}\left(\gamma, \beta^{*}\right)$ as follows. Suppose that $R\left(\gamma, \beta^{*}\right)$ is the $\beta^{*}$ th countably infinite subset of $\bigcup_{\gamma^{\prime}<\gamma} X_{\gamma^{\prime}}$ in that list and $R\left(\gamma, \beta^{*}\right)$ has the property that $\left\{l(r): r \in R\left(\gamma, \beta^{*}\right)\right\} \nearrow \gamma$. (This notation means that every neighborhood of $\gamma$ with respect to the order topology on $\alpha$ 
contains $l(r)$ except for finitely many $r$.) Then we represent $R\left(\gamma, \beta^{*}\right)$ as the disjoint union of two sets $R^{p}\left(\gamma, \beta^{*}\right)$ and $R^{q}\left(\gamma, \beta^{*}\right)$ having the latter property. If $\beta^{*}$ does not belong to the index set of the list or if $\left\{l(r): r \in R\left(\gamma, \beta^{*}\right)\right\} \nearrow \gamma$ is not satisfied, we set $R^{p}\left(\gamma, \beta^{*}\right)=$ $R^{q}\left(\gamma, \beta^{*}\right)=\varnothing$.

We are now ready to describe the points of the level set $X_{\beta}(\beta \neq 0, \beta<\alpha)$ and their basic neighborhoods in $\bigcup_{\gamma^{\prime} \leq \beta} X_{\gamma^{\prime}}$.

First determine $\delta<\alpha$ such that $\beta=\sum_{\gamma<\delta}\left(\lambda_{\gamma}\right)^{\omega}+\beta^{*}$ with $\beta^{*} \in\left(\lambda_{\delta}\right)^{\omega}$.

Note that $\beta \geq \delta \geq 1$.

Case 1. Suppose that $\delta$ is a successor ordinal.

By the induction hypothesis we can suppose that we have prepared a well-ordered list of $\left[X_{\delta-1}\right]^{\omega}$, where we have used an initial segment of $\left\{\epsilon \in O n: 0<\epsilon<\left(\lambda_{\delta}\right)^{\omega}\right\}$ as index set of this list.

Consider any open set $U$ in $\bigcup_{\mu<\beta} X_{\mu}$ such that $U \backslash F$ is of height $\beta$ for any finite set $F$. For any such open set $U$, we put two points $p_{U}$ and $q_{U}$ into the level set $X_{\beta}$. The basic neighborhoods of $p_{U}$ are $\left[\left\{p_{U}\right\} \cup U \cup R_{*}^{p}\left(\delta, \beta^{*}\right)\right] \backslash F$, where $F$ is a finite set. Similarly, the basic neighborhoods for $q_{U}$ are $\left[\left\{q_{U}\right\} \cup U \cup R_{*}^{q}\left(\delta, \beta^{*}\right)\right] \backslash F$, where $F$ is a finite set. Here for $i \in\{p, q\}, R_{*}^{i}\left(\delta, \beta^{*}\right)$ is an open neighborhood of $R^{i}\left(\delta, \beta^{*}\right)$ in $\bigcup_{\gamma<\delta} X_{\gamma}$. If it is possible, choose this open neighborhood such that $\left[R_{*}^{i}\left(\delta, \beta^{*}\right) \backslash R^{i}\left(\delta, \beta^{*}\right)\right] \subseteq U$. (In fact, we shall show in Lemma 8 below that such a choice is always possible. Note that our notation does not indicate that these neighborhoods depend on $U$.)

Case 2. Suppose that $\delta$ is a limit ordinal.

Since $\beta \geq \delta$, we can assume that we have prepared a well-ordered list of $\left[\bigcup_{\gamma<\delta} X_{\gamma}\right]^{\omega}$ as described above.

Consider any open set $U$ in $\bigcup_{\mu<\beta} X_{\mu}$ such that $U \backslash F$ is of height $\beta$ whenever $F$ is a finite set. For any such $U$ put two points $p_{U}$ and $q_{U}$ into $X_{\beta}$. The basic neighborhoods are $\left[\left\{p_{U}\right\} \cup U \cup R_{*}^{p}\left(\delta, \beta^{*}\right)\right] \backslash F$ for $p_{U}$, and $\left[\left\{q_{U}\right\} \cup U \cup R_{*}^{q}\left(\delta, \beta^{*}\right)\right] \backslash F$ for $q_{U}$, respectively. In either case $F$ denotes a finite set. Furthermore for $i \in\{p, q\}, R_{*}^{i}\left(\delta, \beta^{*}\right)$ is an open neighborhood of $R^{i}\left(\delta, \beta^{*}\right)$ in $\bigcup_{\gamma<\delta} X_{\gamma}$. If it is possible, we choose this neighborhood in a way that $\left[R_{*}^{i}\left(\delta, \beta^{*}\right) \backslash R^{i}\left(\delta, \beta^{*}\right)\right] \subseteq U$. (It will follow from Lemma 8 below that such a neighborhood can always be found. Again our notation does not indicate that the chosen neighborhood depends on $U$.)

This concludes the construction of the space $\bigcup_{\gamma \leq \beta} X_{\gamma}$.

During the induction we create new well-ordered lists etc. in the way described above whenever the sets are available to do so.

Finally, let $X_{\alpha}$ consist of two additional points $\infty$ and $-\infty$. A neighborhood of such a point $x$ is a cofinite subset of $X=\bigcup_{\gamma \leq \alpha} X_{\gamma}$ containing $x$.

Note that for any $\beta \leq \alpha, X_{\beta}$ really consists of the isolated points of $X \backslash \bigcup_{\gamma<\beta} X_{\gamma}$.

We next verify that the example is well defined; i.e. the indexing of the lists is possible.

LemMa 2. The induction was indexed correctly. 
Proof. The construction above works under the assumption that $\left|X_{\gamma}\right| \leq \lambda_{\gamma+1}$ for any ordinal $\gamma<\alpha$, and that $\left|\bigcup_{\beta<\gamma} X_{\beta}\right| \leq \lambda_{\gamma}$ for any limit ordinal $\gamma<\alpha$. Thus it is clearly sufficient to show that $\left|X_{\beta}\right| \leq \lambda_{\beta+1}$ for any ordinal $\beta<\alpha$.

This holds for $\beta=0$. Suppose that $\beta<\alpha, \beta \neq 0$ and that the inequality $\left|X_{\beta^{\prime}}\right| \leq \lambda_{\beta^{\prime}+1}$ holds for all $\beta^{\prime}<\beta$. Assume that $\beta$ is a limit ordinal. By the inductive assumption, $\bigcup_{\beta^{\prime}<\beta} X_{\beta^{\prime}}$ has cardinality at most $\sum_{\beta^{\prime}<\beta} \lambda_{\beta^{\prime}+1}$. Therefore, by the construction of the space, we conclude that $\left|X_{\beta}\right| \leq 2^{\Sigma_{\beta^{\prime}<\beta} \lambda_{\beta^{\prime}}} \leq \lambda_{\beta+1}$.

If $\beta$ is a successor ordinal, an analogous argument shows that $\left|X_{\beta}\right| \leq \lambda_{\beta+1}$, too.

We shall now prove several lemmas in order to verify the assertion on neighborhoods made during the construction of the space.

Lemma 3. Let $U$ be an open subset of $X$ of height greater than $\beta$ and $\beta=\sum_{\gamma<\delta}\left(\lambda_{\gamma}\right)^{\omega}+\beta^{*}$, where $\beta^{*} \in\left(\lambda_{\delta}\right)^{\omega}$ and $\delta<\alpha$. Then $U$ contains an infinite subset of $R\left(\delta, \beta^{*}\right)$ provided that the set $R^{p}\left(\delta, \beta^{*}\right) \neq \varnothing$.

Proof. The set $U$ contains a point of the level set $X_{\beta}$. By the construction of the basic neighborhoods of this point, an infinite subset of $R\left(\delta, \beta^{*}\right)$ must be contained in $U$ provided that the set $R^{p}\left(\delta, \beta^{*}\right)$ is nonempty.

LEMMA 4. Let $U$ be an open subset of $X$ of height greater than $\xi, \xi=\sum_{\gamma^{\prime}<\delta}\left(\lambda_{\gamma^{\prime}}\right)^{\omega}$ and $\gamma<\delta<\alpha$. Then $U$ contains a cofinite subset of $X_{\kappa}$, for any $\kappa<\gamma$.

Proof. Apply Lemma 3 to each $\beta=\sum_{\gamma^{\prime}<\kappa+1}\left(\lambda_{\gamma^{\prime}}\right)^{\omega}+\beta^{*}$, where $\beta^{*} \in\left(\lambda_{\kappa+1}\right)^{\omega}$. Since $U$ has height greater than $\beta, U$ contains an infinite subset of $R\left(\kappa+1, \beta^{*}\right)$ for all such $\beta^{*}$ for which the set $R^{p}\left(\kappa+1, \beta^{*}\right)$ is nonempty. Therefore $U$ contains an infinite subset of every countably infinite subset of $X_{\kappa}$. We conclude that $U$ contains a cofinite subset of $X_{\kappa}$.

LEMMA 5. Let $U$ be an open subset of $X$ of height greater than $\xi, \xi=\sum_{\gamma^{\prime}<\delta}\left(\lambda_{\gamma^{\prime}}\right)^{\omega}$ and $\gamma$ is a limit ordinal with $\gamma<\delta<\alpha$. Then $U$ contains all but a finite set of each countably infinite set $R \subseteq \bigcup_{\gamma^{\prime}<\gamma} X_{\gamma^{\prime}}$ such that $\{l(r): r \in R\} \nearrow \gamma$.

Proof. We can apply Lemma 3 to each $\beta=\sum_{\gamma^{\prime}<\gamma}\left(\lambda_{\gamma^{\prime}}\right)^{\omega}+\beta^{*}$, where $\beta^{*} \in\left(\lambda_{\gamma}\right)^{\omega}$, because $U$ has height greater than $\beta$. Then $U$ contains an infinite set of $R\left(\gamma, \beta^{*}\right)$ for all $\beta^{*}$ such that $R^{p}\left(\gamma, \beta^{*}\right)$ is nonempty. Consequently $U$ contains an infinite subset of each countably infinite subset $R \subseteq \bigcup_{\gamma^{\prime}<\gamma} X_{\gamma^{\prime}}$ having the property that $\{l(r): r \in R\} \nearrow \gamma$. We conclude that $U$ contains a cofinite subset of each such $R$.

LEMMA 6. Let $U$ be an open subset of $X$ having height greater than $\xi, \xi=\sum_{\gamma^{\prime}<\delta}\left(\lambda_{\gamma^{\prime}}\right)^{\omega}$ and $\gamma<\delta<\alpha$. Then $U$ contains a cofinite subset of $\bigcup_{\gamma^{\prime}<\gamma} X_{\gamma^{\prime}}$. 
Proof. If $U$ misses a countably infinite subset $S$ of $\bigcup_{\gamma^{\prime}<\gamma} X_{\gamma^{\prime}}$, then there exists $\gamma^{\prime}<\gamma$ such that $\left|S \cap X_{\gamma^{\prime}}\right|=\omega$, or there exist $\gamma^{\prime} \leq \gamma$ and a countably infinite set $R \subseteq S \cap \bigcup_{\kappa<\gamma^{\prime}} X_{\kappa}$ having the property that $\{l(r): r \in R\} \nearrow \gamma^{\prime}$.

In the first case, we obtain a contradiction, because, by Lemma $4, U$ contains a cofinite subset of $X_{\gamma}$. In the second case, we see that, by Lemma 5, $U$ contains a cofinite subset of $R$. Again we have reached a contradiction. of $X$.

Lemma 7. Let $U$ be an open subset of $X$ having height $\alpha$. Then $U$ is a cofinite subset

Proof. If $U$ misses a countably infinite subset $A$ of $X \backslash\{-\infty, \infty\}$, then since $\operatorname{cf}(\alpha)>\omega$, there exists $\gamma<\alpha$ such that $A \subseteq \bigcup_{\gamma^{\prime}<\gamma} X_{\gamma^{\prime}}$. We apply now Lemma 6 to $\delta=\gamma+1$. Since $\xi:=\sum_{\gamma^{\prime}<\delta}\left(\lambda_{\gamma^{\prime}}\right)^{\omega}<\sum_{\gamma^{\prime}<\alpha}\left(\lambda_{\gamma^{\prime}}\right)^{\omega}=\alpha$, the set $U$ has height greater than $\xi$. Therefore $U$ contains a cofinite subset of $\bigcup_{\gamma^{\prime<\gamma}} X_{\gamma^{\prime}}-$ a contradiction.

LEMMA 8. Let $U$ be an open subset of $X$ such that for any finite set $F, U \backslash F$ is of height $\beta<\alpha$, where $\beta \neq 0, \beta=\sum_{\gamma^{\prime}<\delta}\left(\lambda_{\gamma^{\prime}}\right)^{\omega}+\beta^{*}, \beta^{*} \in\left(\lambda_{\delta}\right)^{\omega}$ and $\delta<\alpha$.

When constructing the space $X$ we can always find for $i \in\{p, q\}$ an open neighborhood $R_{*}^{i}\left(\delta, \beta^{*}\right)$ of $R^{i}\left(\delta, \beta^{*}\right)$ in $\bigcup_{\gamma<\delta} X_{\gamma}$ such that $\left[R_{*}^{i}\left(\delta, \beta^{*}\right) \backslash R^{i}\left(\delta, \beta^{*}\right)\right] \subseteq U$.

Proof. The statement is nontrivial only for those $R^{i}\left(\delta, \beta^{*}\right) \neq \varnothing$ for which $\delta \neq 1$, since otherwise $R^{i}\left(\delta, \beta^{*}\right)$ is open. Hence we consider this situation. (Note that then in particular $\beta^{*} \neq 0$.)

Case 1. Suppose first that $\delta \neq 1$ is a successor ordinal. Applying Lemma 6 for $\gamma=\delta-1$, we see that the set $U$ contains all but a finite subset $E$ of $\bigcup_{\gamma^{\prime}<\gamma} X_{\gamma^{\prime}}$, because $U$ has height $\beta=\sum_{\gamma^{\prime}<\delta}\left(\lambda_{\gamma^{\prime}}\right)^{\omega}+\beta^{*}>\sum_{\gamma^{\prime}<\delta}\left(\lambda_{\gamma^{\prime}}\right)^{\omega}$. By definition, $R^{i}\left(\delta, \beta^{*}\right) \subseteq X_{\gamma^{\prime}}$. Since $X$ is scattered, by using the $T_{1}$-property we can clearly find an open neighborhood $R_{*}^{i}\left(\delta, \beta^{*}\right)$ of $R^{i}\left(\delta, \beta^{*}\right)$ in $\bigcup_{\gamma^{\prime} \leq \gamma} X_{\gamma^{\prime}}$ such that $R_{*}^{i}\left(\delta, \beta^{*}\right) \backslash R^{i}\left(\delta, \beta^{*}\right)$ is contained in $U$, because $E$ is finite.

Case 2. Suppose now that $\delta$ is a limit ordinal. Set $E:=\left(\bigcup_{\gamma^{\prime}<\delta} X_{\gamma^{\prime}}\right) \backslash U$. By Lemma 6 there cannot exist $\gamma<\delta$ such that $E \cap \bigcup_{\gamma^{\prime}<\gamma} X_{\gamma^{\prime}}$ is infinite, since the set $U$ has height $\beta=\sum_{\gamma^{\prime}<\delta}\left(\lambda_{\gamma^{\prime}}\right)^{\omega}+\beta^{*}>\sum_{\gamma^{\prime}<\delta}\left(\lambda_{\gamma^{\prime}}\right)^{\omega}$. Hence $E$ must be equal to a countably infinite set $R$ such that $\{l(r): r \in R\} \nearrow \delta$ or $E$ must be finite. In either case by the $T_{1}$-property and scatteredness of $X$ we can then clearly find an open neighborhood $R_{*}^{i}\left(\delta, \beta^{*}\right)$ of $R^{i}\left(\delta, \beta^{*}\right) \nearrow \delta$ in $\bigcup_{\gamma^{\prime}<\delta} X_{\gamma^{\prime}}$ such that $\left[R_{*}^{i}\left(\delta, \beta^{*}\right) \backslash R^{i}\left(\delta, \beta^{*}\right)\right]$ is a subset of $U$.

Proposition 2. The space $X$ constructed above is a $T_{1}$-space in which each open set is the intersection of two compact open sets. (Hence by the criterion cited in the introduction it admits a unique quasi-proximity.) Nevertheless $X$ is not hereditarily compact.

Proof. As we have already observed, $X$ is clearly a $T_{1}$-space by construction. Since it has infinitely many isolated points, it is not hereditarily compact. By Lemma 7 each open 
set $U$ of height $\alpha$ is cofinite in $X$. Thus $U=(U \cup\{\infty\}) \cap(U \cup\{-\infty\})$, where the two latter sets are compact and open. If $U$ is an open set of $X$ of height $\alpha+1$, then $\infty$ or $-\infty$ belong to $U$. Thus clearly $U$ is cofinite and compact.

Let $U$ be an open subset of $X$ of height $\beta<\alpha, \beta \neq 0$ and $\beta=\sum_{\gamma^{\prime}<\delta}\left(\lambda_{\gamma^{\prime}}\right)^{\omega}+\beta^{*}$, where $\beta^{*} \in\left(\lambda_{\delta}\right)^{\omega}$ and $\delta<\alpha$. If $U \backslash F$ has height $\beta$ whenever $F$ is a finite set, then, by Lemma $8, U$ can be written as $\left[\left\{p_{U}\right\} \cup U \cup R_{*}^{p}\left(\delta, \beta^{*}\right)\right] \cap\left[\left\{q_{U}\right\} \cup U \cup R_{*}^{q}\left(\delta, \beta^{*}\right)\right]$, where $p_{U}$ and $q_{U}$ belong to $X_{\beta}$ and either of the unions on the right hand side is compact and open.

On the other hand, if there is a finite set $F$ such that the height of $U \backslash F$ is strictly smaller than $U$, then there exists a finite subset $E$ of $U$ such that the height of $U \backslash E$ is equal to the height of $(U \backslash E) \backslash F$ whenever $F$ is a finite set. Choose for each $e \in E$ an open neighborhood $U(e)$ such that $U(e) \subseteq U$. In the light of the preceding subcase it is clear that the open set $U \backslash E$ can be written as the intersection of two compact open sets, say $G_{1}$ and $G_{2}$. Then $U=\left(\bigcup_{e \in E} U(e) \cup G_{1}\right) \cap\left(\bigcup_{e \in E} U(e) \cup G_{2}\right)$ and we conclude that $U$ is the intersection of two compact open sets.

We finally remark that $X$ is sober, since each scattered space is sober. (A topological space is called sober provided that each closed irreducible subspace is the closure of a unique point, where a nonempty topological space is said to be irreducible provided that any pair of nonempty open sets intersects.) Note also that $X$ is locally compact; i.e. each point has a base consisting of compact neighborhoods. In fact, by construction $X$ has a base that consists of compact open sets.

Let us mention that our work on the question dealt with above can be considered a possible starting point of general investigations on the problem of how a topology is obtained from its bases. Suppose that we are given a (possibly finite) sequence $s=\left(s_{\alpha}\right)_{\alpha<\beta}$ of cardinals larger than 2. Assume furthermore that all odd entries of this sequence are at most $\omega$. Let us denote by $s$-space any topological space $X$ which has the property that for each base $\mathscr{B}$ of $X, \bigcup_{\alpha<\beta} \mathscr{B}_{\alpha}$ is the topology of $X$, where $\mathscr{B}_{-1}=\mathscr{B}$ and for any ordinal $\gamma$ such that $\gamma<\beta$, the collection $\mathscr{B}_{\gamma}$ is obtained from $\bigcup_{\alpha<\gamma} \mathscr{B}_{\alpha}$ by taking unions (resp. intersections) of fewer than $s_{\gamma}$ elements in $\bigcup_{\alpha<\gamma} \mathscr{B}_{\alpha}$ provided that $\gamma$ is even (resp. odd). Note that using this terminology each hereditarily compact space is an $(\omega)$-space, the space constructed above is an $(\omega, 3)$-space and uqp-spaces can be re-defined as $(\omega, \omega)$-spaces. De Morgan's laws show that distinct sequences $s$ can yield the same class of spaces; e.g. uqp spaces can also be defined as $s_{0}$-spaces, where $s_{0}$ denotes the $\omega$-sequence all entries of which are $\omega$.

The example given above suggests the conjecture that whenever two sequences $s$ and $t$ yield distinct classes of spaces, then the intersections of these two classes with the class of $T_{1}$-spaces will differ, too.

\section{REFERENCES}

1. G. C. L. Brümmer and H. P. A. Künzi, Sobrification and bicompletion of totally bounded quasi-uniform spaces, Math. Proc. Camb. Phil. Soc. 101 (1987), 237-247.

2. J. Ferrer, On topological spaces with a unique quasi-proximity, Quaestiones Math. 17 (1994), $479-486$. 
3. P. Fletcher and W. F. Lindgren, Quasi-uniform spaces (Dekker, 1982).

4. H. P. A. Künzi, Topological spaces with a unique compatible quasi-proximity, Arch. Math. 43 (1984), 559-561.

5. H. P. A. Künzi, Some remarks on quasi-uniform spaces, Glasgow Math. J. 31 (1989), 309-320.

6. H. P. A. Künzi, Quasi-uniform spaces-eleven years later, Topology Proc. 18 (1993), 143-171.

7. W. F. Lindgren, Topological spaces with a unique compatible quasi-uniformity, Canad. Math. Bull. 14 (1971), 369-372.

8. W. F. Lindgren, Topological spaces with unique quasi-uniform structure, Arch. Math. 22 (1971), 417-419.

9. A. H. Stone, Hereditarily compact spaces, Amer. J. Math. 82 (1960), 900-916.

Department of Mathematics

UNIVERSITY OF BERNE

SIDLERSTRASSE 5

CH-3012 BERNE

SWITZERLAND

E-mail:kunzi@math-stat.unibe.ch
Department of Mathematics

YORK UNIVERSITY

NORTH YORK

ONTARIO

Canada M3J 1P3

E-mail: stephen.watson@mathstat.yorku.ca. 